\title{
Enriched Early Life Experiences Reduce Adult Anxiety-Like Behavior in Rats: A Role for Insulin-Like Growth Factor 1
}

\author{
Sara Baldini, ${ }^{1}$ Laura Restani, ${ }^{2}$ Laura Baroncelli, ${ }^{2}$ Maila Coltelli, ${ }^{3}$ Roberta Franco, ${ }^{3}$ Maria Cristina Cenni, ${ }^{2}$ \\ Lamberto Maffei, ${ }^{1,2}$ and Nicoletta Berardi ${ }^{2,3}$ \\ ${ }^{1}$ Laboratory of Neurobiology, Scuola Normale Superiore, I-56126 Pisa, Italy, ${ }^{2}$ Institute of Neuroscience, National Research Council, I-56124 Pisa, Italy, and \\ ${ }^{3}$ Department of Neuroscience, Psychology, Drug Research, and Child Health, University of Florence, I-50135 Florence, Italy
}

Early life experiences can affect brain development, contributing to shape interindividual differences in stress vulnerability and anxiety-like behavior. In rodents, high levels of maternal care have long-lasting positive effects on the behavior of the offspring and stress response; postweaning rearing in an enriched environment (EE) or massage counteract the negative effects of maternal separation or prenatal stressors. We recently found that insulin-like growth factor 1 (IGF-1) is a key mediator of early EE or massage on brain development. Whether early enrichment of experience can induce long-lasting effects on anxiety-like behavior and whether IGF-1 is involved in these effects is not known. We assessed anxiety-like behavior by means of the elevated plus maze in control adult rats and in adult rats subjected to early EE or to massage. We found that both $\mathrm{EE}$ and massage reduced adult anxiety-like behavior. Early IGF-1 systemic injections in rat pups reared in standard condition mimic the effects of EE and massage, reducing anxiety-like behavior in the adult; blocking early IGF-1 action in massaged and EE animals prevents massage and EE effects. In EE and IGF-1-treated animals, we assessed the hippocampal expression of glucocorticoid receptors (GRs) at postnatal day 12 (P12) and P60, finding a significantly higher GR expression at P60 for both treatments. These results suggest that IGF-1 could be involved in mediating the long-lasting effects of early life experiences on vulnerability/resilience to stress in adults.

\section{Introduction}

Early life experiences can affect brain development, contributing to shape interindividual differences in vulnerability to stress and anxiety-like behavior. The molecular mechanisms underlying these effects are under active investigation. In rats, several studies have underlined that variations in maternal care levels induce long-lasting effects on the behavior of the offspring, on hippocampal structure and function, and on stress responses (Levine, 1967; for review, see Meaney and Szyf, 2005; Zhang et al., 2013). In particular, offspring of mothers displaying high level of licking and grooming show, once adult, increased exploratory behavior and spatial memory, reduced anxiety-like behavior, and a higher level of glucocorticoid receptors (GRs) in the hippocampus, leading to a difference in the feedback control on hypothalamus-pituitary-adrenal (HPA) axis and to differences in spine density and synaptic plasticity in the hippocampus (Liu et al.,1997, 2000; Zhang et al., 2013).

Enriched environment (EE), either after weaning or including the post-weaning period, is effective in protecting from or revers-

\footnotetext{
Received July 24, 2012; revised April 30, 2013; accepted June 8, 2013.

Author contributions: S.B., L.M., and N.B. designed research; S.B., L.R., L.B., M.C., R.F., and M.C.C. performed research; S.B., L.R., L.B., M.C.C., and N.B. analyzed data; S.B., L.R., L.B., M.C.C., L.M., and N.B. wrote the paper.

This work was supported by Research Programs of Relevant National Interest/Ministry of Education, Universities, and Research funding (L.M.). We thank Profs. Tommaso Pizzorusso and Matteo Caleo for helpful discussions and critical reading of this manuscript.

Correspondence should be addressed to Nicoletta Berardi, Institute of Neuroscience, National Research Council, Via G. Moruzzi 1, I-56124 Pisa, Italy. E-mail: berardi@in.cnr.it.

S. Baldini's present address: Department of Developmental Neuroscience, Stella Maris Scientific Institute, Viale del Tirreno 331, I-56128 Calambrone, Pisa, Italy.

DOI:10.1523/JNEUROSCI.3541-12.2013

Copyright $\odot 2013$ the authors $\quad 0270-6474 / 13 / 3311715-09 \$ 15.00 / 0$
}

ing the effect of a stressor in animals that are highly emotional or anxious (Chapillon et al., 2002; Francis et al., 2002; Laviola et al., 2004). EE in adult rats induces higher expression of the gene encoding GRs in the hippocampus (Mohammed et al., 1993; Olsson et al., 1994). Protocols of massage, which mimics maternal licking and grooming and might be thought of as an early enrichment of experience, rescue the negative effects of maternal separation/deprivation or of prenatal stressors on pup growth, growth hormone secretion, HPA axis, brain-derived neurotrophic factor (BDNF), and synaptophysin expression (Schanberg and Field, 1987; Chatterjee et al., 2007).

We recently found that early EE or massage affect brain development and that insulin-like growth factor 1 (IGF-1) is a key mediator of these effects (Ciucci et al., 2007; Guzzetta et al., 2009; Landi et al., 2009; Sale et al., 2009). Higher IGF-1 levels are found in massaged (MSG) preterm infants (Field et al., 2008; Guzzetta et al., 2009) and in early MSG and EE rat pups (Ciucci et al., 2007; Sale et al., 2007; Guzzetta et al., 2009); antagonizing circulating IGF-1 blocks massage effects on visual acuity development in rats (Guzzetta et al., 2009).

Whether early enrichment of experience by pre-weaning EE or massage can induce long-lasting effects on anxiety-like behavior and whether IGF-1 is involved in these effects are not known.

We found that both massage and EE reduced adult anxietylike behavior. Early IGF-1 systemic injections mimicked, whereas blocking early IGF-1 action prevented, the effects of massage and EE on adult anxiety-like behavior. To investigate the molecular mechanisms underlying the reduction of adult anxiety after early enrichment of experience and IGF-1 early treatment, we assessed the hippocampal expression of GRs in EE and IGF-1-treated an- 
imals at both postnatal day 12 (P12) and P60. We found a significant increase in GR expression at P60 for both treatments. This suggests that IGF-1 triggers the strengthening of negative feedback in the HPA axis found in enriched animals.

\section{Materials and Methods}

A total of 193 Long-Evans hooded rats from 40 litters were used in this study, approved by the Italian Ministry of Public Health and in line with guidelines for care and use of laboratory animals. We used both male and female rats (94 males and 99 females). Females and males were equally distributed among experimental groups, with the maximum discrepancy being $60 \%$ male and $40 \%$ females or vice versa. Animals in each experimental group always came from different litters, with a minimum of three litters even for the smallest groups, to prevent litter effects from occurring.

\section{Animal treatment}

Female rats were put with males (one male for every mating cage) in standard cages for reproduction $(60 \times 40 \times 20 \mathrm{~cm})$. Parturition was checked one time a day, and the day of birth was considered P0. To identify individual newborn rats in each litter, we cut the outermost phalanx in fingers from the left or right forepaw.

Environmental enrichment. Pregnant female rats were assigned to either EE or standard rearing conditions [control (CNT)] at least $7 \mathrm{~d}$ before delivery. We used an EE protocol as described by Ciucci et al. (2007), and we designed three experimental groups: (1) EE up to P12 (EE-P12); (2) EE-P45; and (3) EE-P60. At the end of each EE condition, the animals were relocated in standard laboratory cages $(30 \times 40 \times 20 \mathrm{~cm})$. For the CNT condition, dams with their offspring live in standard laboratory cages. Litter and food were the same in all environmental conditions.

Massage protocol. We used the same massage protocol as in the study by Guzzetta et al. (2009). Briefly, two groups of rat pups living in standard cages were used: (1) one group (MSG group) was separated from the mother to receive tactile stimulation three times per day from $\mathrm{P} 1$ to $\mathrm{P} 12$; and (2) the other group was left undisturbed with the mother (CNT group). The stimulation protocol consisted in $5 \mathrm{~min}$ of tactile stimulation that combined gently stroking and massaging to mimic maternal care. At the end of each session, animals were replaced with their mother.

Subcutaneous injections. Injections of human IGF-1 (IBT Systems) were performed subcutaneously in non-enriched pups as in the study by Landi et al. (2009) (IGF-1 group) from P1 to P9. For each pup, a single injection per day was performed at 9:00 A.M.; IGF-1 concentration was $10 \mu \mathrm{g} / \mu \mathrm{l}$, and the volume of the injection was adjusted to the pup weight to reach a dose of $1.8 \mu \mathrm{g} / \mathrm{g}$ body weight, as in the study by Landi et al. (2009). A group of pups was treated with subcutaneous injections of saline as controls [vehicle-treated (Veh) group].

Injections of JB1 (Bachem) were performed subcutaneously in MSG pups and in EE pups from P1 to P9 as in the study by Guzzetta et al. (2009). Control MSG and EE pups were treated with subcutaneous injections of saline (MSG or EE plus Veh). JB1 is an IGF-1 peptide analog that specifically and potently inhibits the autophosphorylation of the IGF-1 receptor (IGF$1 \mathrm{R})$ by IGF-1 and is primarily used, both intracerebroventricularly and subcutaneously, to block IGF-1 action and to prevent systemic IGF-1 from crossing the blood-brain barrier (Fernandez et al., 1997; Carro et al., 2000, Malberg et al., 2007; Hoshaw et al., 2008; Guzzetta et al., 2009; Brock et al., 2011; Witty et al., 2013). In particular, Guzzetta et al. (2009) and Brock et al. (2011) used a subcutaneous protocol of JB1 administration in pups of the same age as those used in our work.

Post-weaning housing. For all experimental groups, rat pups were separated from their mother at weaning age (P22). For animals that were in standard environment at the moment of weaning (groups: controls, EE-P12 and massage), we kept them in groups of three to four animals per cage; for groups that were in $\mathrm{EE}$ at the moment of weaning (groups: EE-P45 and EE-P60), we left them in EE, taking away the mother.

\section{Behavioral test: elevated plus maze}

All EE, MSG, IGF-1-treated, JB1-treated, Veh, and CNT rats were tested for anxiety responses at $\mathrm{P} 60$ by means of the elevated plus maze (EPM). Behavioral assessments were conducted in a dedicated room different from where animals were housed. The range of results for our CNT animals are in the range reported for young adult Long-Evans rats in the literature (Chee and Menard, 2011; Trent and Menard, 2011; Hayton et al., 2012; Wilkin et al., 2012).

The maze is made of black polyvinyl chloride and consists of four arms, $50 \mathrm{~cm}$ long $\times 10 \mathrm{~cm}$ wide, connected by a central square, $10 \times 10$ $\mathrm{cm}$ : two open without walls and two closed by $31-\mathrm{cm}$-high walls. All arms are attached to sturdy plastic legs; the maze is elevated $55 \mathrm{~cm}$ above the floor level and is set in a dimly lit room; a video camera is suspended above the maze to record the rat movements for analysis. A videotracking system (Ethovision System) is used to automatically collect behavioral data. The procedure consists of placing the rats at the junction of the open and closed arms, the center of the maze, facing the open arm opposite to where the experimenter is. The video-tracking system is started after the animal is placed in the maze so that the behavior of each animal is consistently recorded for $5 \mathrm{~min}$. At the end of the $5 \mathrm{~min}$ test, the rat is removed from the plus maze and returned to its home cage. The experiments were performed from 9:00 A.M. to 12:00 P.M. All behavioral assessments were performed blind to the treatments.

\section{Western blot}

Brains were rapidly removed, and hippocampi were dissected and frozen on dry ice. All tissue dissections were performed from 10:00 A.M. to 12:00 P.M. Proteins were extracted with lysis buffer (20 mM Tris- $\mathrm{HCl}, \mathrm{pH}$ 7.45, $150 \mathrm{~mm} \mathrm{NaCl}, 10 \mathrm{~mm}$ EDTA, $0.1 \mathrm{~mm} \mathrm{Na}_{3} \mathrm{VO}_{4}, 1 \mathrm{~mm}$ PMSF, $1 \mu \mathrm{g} / \mathrm{ml}$ leupeptin, $1 \mu \mathrm{g} / \mathrm{ml}$ aprotinin, $1 \%$ Triton X-100, and $10 \%$ glycerol), and total concentration of the samples was assessed with a protein assay kit (Bio-Rad) using a bovine serum albumin-based standard curve. Protein extracts of each sample $(50 \mu \mathrm{g})$ were separated by electrophoresis and blotted; filters were blocked and incubated overnight at $4^{\circ} \mathrm{C}$ with primary antibodies (anti-IGF-1R at 1:500 dilution, anti-GR at 1:1000, and antiBDNF at 1:500; all from Santa Cruz Biotechnology). Filters were also probed with anti- $\alpha$-tubulin antibody (polyclonal, 1:15,000 dilution; Abcam) as an internal standard for protein quantification. Blots were then rinsed [0.2\% Tween 20 added to PBS (TPBS)], incubated in infrared labeled secondary antibodies (anti-rabbit IRDye 680LT at 1:30,000 or anti-rabbit $800 \mathrm{CW}$ at 1:20,000; Li-Cor Biosciences), washed in TPBS, and briefly rinsed in PBS. Filters were scanned using an Odyssey IR scanner (Li-Cor Biosciences), and densitometry analysis was performed with Image Studio software version 3.1 (Li-Cor Biosciences). The band of interest was determined with respect to protein molecular weight standards or to control peptide. Antibody signal was calculated as integrated intensity of the region defined around the band of interest. Protein amount of IGF-1R, GR, and BDNF was normalized on $\alpha$-tubulin signal.

\section{Immunohistochemistry for NeuN}

Animals were deeply anesthetized and perfused via intracardial infusion with $0.1 \mathrm{M}$ PBS and then 4\% paraformaldehyde (PFA; dissolved in $0.1 \mathrm{M}$ phosphate buffer, $\mathrm{pH}$ 7.4). Brains were removed, fixed overnight in PFA, transferred to $30 \%$ sucrose solution, and stored at $4^{\circ} \mathrm{C}$. Coronal sections were cut at $40 \mu \mathrm{m}$ thickness on a freezing microtome. After a blocking step (10\% BSA, $0.3 \%$ Triton X-100 in PBS), free-floating sections were incubated overnight at $4^{\circ} \mathrm{C}$ with the mouse monoclonal NeuN antibody (1:500; Millipore Bioscience Research Reagents); antigen-antibody binding was then revealed with goat anti-mouse IgG conjugated to Alexa Fluor 568 fluorophore (1:400; Invitrogen). Slices were mounted on glass slides and covered with VectaShield mounting medium (Vector Laboratories). Images from the dorsal hippocampus (dCA1-dCA3 and DG), amygdala, and the binocular portion of the primary visual cortex were acquired at $40 \times$ magnification, zoom $1 \times$, using a Leica confocal microscope (numerical aperture 1.25; field, $375 \times 375 \mu \mathrm{m}$ acquired at $1024 \times 1024$ pixels). Settings for laser intensity, gain, offset, and pinhole were optimized initially and held constant through the experiment. The collected images were imported to the image analysis system MetaMorph (Molecular Devices) to quantify the number of NeuNpositive cells. For each animal, two or three sections per structure were analyzed. Counts were done on the entire thickness of structures.

\section{Statistical analyses}

All statistical analysis was done using SigmaStat (Systat Software). Differences between two groups were assessed with a two-tailed $t$ test for data normally distributed and with Mann-Whitney rank-sum test for 
A

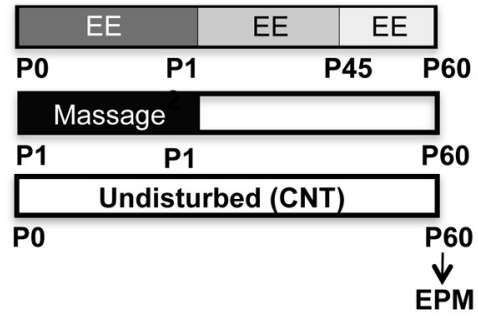

C

Open Arm Time

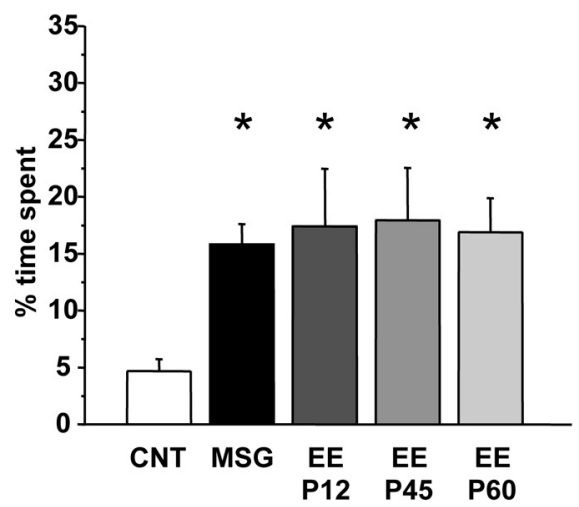

E

Total entries

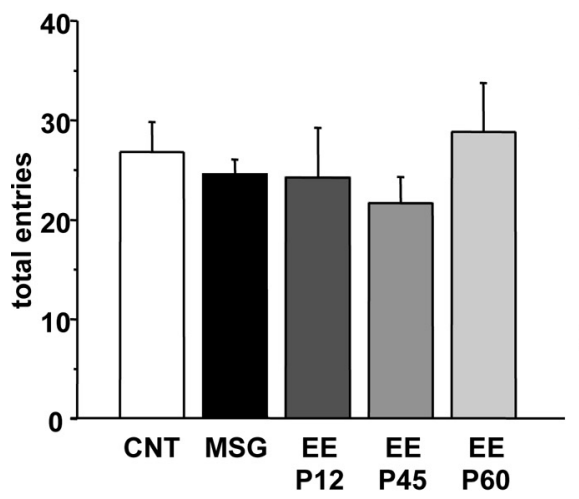

B Examples of movement tracking in P60 rats

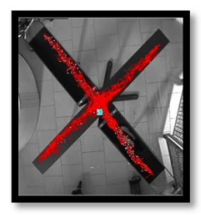

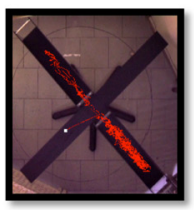

CNT
EE

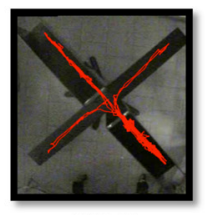

MSG

D

Open Arm Entries

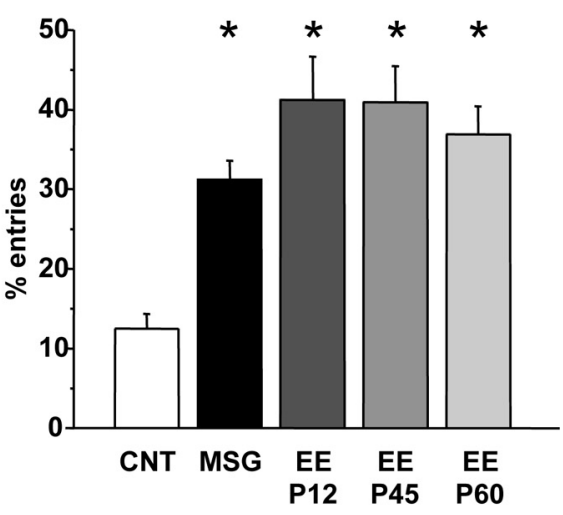

$\mathbf{F}$

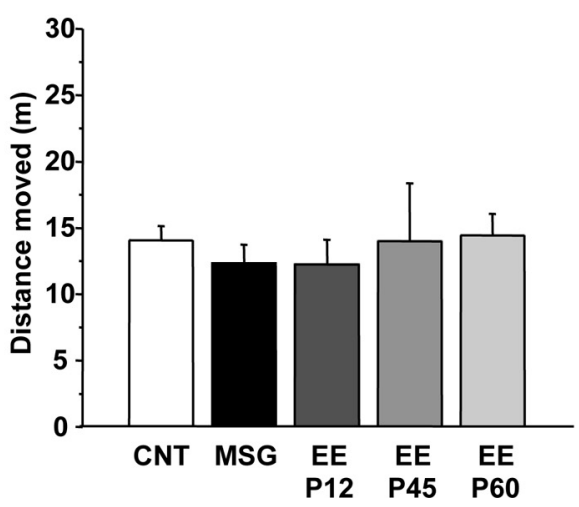

Figure 1. Early postnatal enriched experience is sufficient to affect adult anxiety-like behavior. $\boldsymbol{A}$, Experimental protocols. $\boldsymbol{B}$, Examples of movement tracking in the EPM for one EE up to P12 animal (EE), one MSG animal, and one CNT animal. C, D, Mean percentage of time spent in the open arms $(\boldsymbol{C})$ and mean percentage of entries in the open arms $(\boldsymbol{D})$ assessed at P60 for CNT, MSG, EE-P12, EE-P45, and EE-P60 rats. All treated groups significantly differ from the CNT group (asterisks, $p<0.01$ ), but they do not differ one from each other (one-way ANOVA). $\boldsymbol{E}, \boldsymbol{F}$, Vertical bars are SEM. Mean total arm entries $(\boldsymbol{E})$ and mean total distance moved $(\boldsymbol{F})$ for all experimental groups. No difference was found between groups (one-way ANOVA). Vertical bars are SEM.

data not normally distributed. Differences between more than two groups were evaluated with one-way ANOVA, followed by Holm-Sidak test for data normally distributed and with Kruskal-Wallis one-way ANOVA (ANOVA on ranks) with Dunn's post hoc test for data not normally distributed. Level of significance was $p<0.05$.

\section{Results}

Pre-weaning massage and EE produce long-term effects on anxiety-like behavior

We first assessed the effects of an EE protocol restricted to the pre-weaning period and of massage treatment on adult anxietylike behavior. MSG $(n=32)$ and EE-P12 $(n=11)$ rats spent significantly more time in, and entered more frequently, the open arms of EPM apparatus (percentage time in open arms, $\mathrm{MSG}=15.83 \pm 1.79, \mathrm{EE}-\mathrm{P} 12=$ $17.42 \pm 5.07$; percentage open arm entries, $\mathrm{MSG}=31.18 \pm 2.41, \mathrm{EE}-\mathrm{P} 12=41.18 \pm$ $5.45)$ with respect to $\mathrm{CNT}$ animals $(n=31$; percentage time in open arms, CNT = $4.68 \pm 1.06$; percentage open arm entries, $\mathrm{CNT}=12.51 \pm 1.85$; Fig. $1 C, D)$; the performance of EE-P12 and MSG animals did not differ from that of rats reared in EE for a longer time, up to $\mathrm{P} 45$ ( $n=11$; percentage time in open arms, EE-P45 = $17.94 \pm 4.59$; percentage open arm entries, EE-P45 = $40.91 \pm 4.56)$ or up to $\mathrm{P} 60$ ( $n=10$; percentage time in open arms, EE-P60 $=16.90 \pm$ 2.98; percentage open arm entries, $\mathrm{EE}-$ P60 $=36.88 \pm 3.55)$, suggesting that early enrichment of postnatal environment is sufficient for programming the adult anxiety response [one-way ANOVA, $\mathrm{df}=4$; percentage time in open arms, $p<0.001(F=$ 6.712); percentage open arms entries, $p<$ $0.001(F=17.671)$, post hoc Holm-Sidak method].

To control that the differences in open arm entries and permanence were not attributable to a global difference in exploratory behavior or locomotor activity, we measured the total number of arm entries and the total distance moved, respectively, finding no significant difference between the experimental groups (Fig. $1 E, F$ ) [one-way ANOVA, $\mathrm{df}=4$; total entries, $p>0.05(F=0.327)$; total distance moved, $p>0.05(F=0.291)]$.

\section{Early IGF-1 subcutaneous}

administration results in decreased anxiety-like behavior in adult rats

IGF-1 increase has been shown to be crucial for EE or massage effects on neural development (Ciucci et al., 2007; Sale et al., 2007; Guzzetta et al., 2009); in particular, early IGF-1 administration mimics EE effects on retinal development and on visual acuity development without affecting gross anatomy or cell density in the brain (Ciucci et al., 2007; Sale et al., 2007). Therefore, we assessed whether systemic subcutaneous injections of IGF-1 from P1 to $\mathrm{P} 9$ in rat pups reared in a standard condition could mimic the effects induced by EE and massage on anxiety-like behavior.

IGF-1-injected rats showed a decreased anxiety-like behavior at P60 (Fig. $2 B, C$ ); indeed, both the percentage of time spent and the percentage of entries in open arms in IGF-1-treated animals $(n=13$; percentage time in open arms, IGF- $1=15.20 \pm 3.58$; percentage open arm entries, IGF-1 $=30.32 \pm 3.02$ ) were significantly higher with respect to vehicle (Veh)-treated ( $n=14$; percentage time in open arms, Veh $=6.55 \pm 2.52$; percentage open arm entries, Veh $=15.58 \pm 3.05)$ or untreated $(n=31)$ rats; the latter two groups did not differ [percentage time spent in open arms, one-way ANOVA on ranks, $\mathrm{df}=2, p<0.01(H=10.009)$, 
A

IGF-1/Neh.

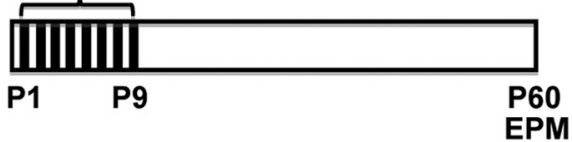

B

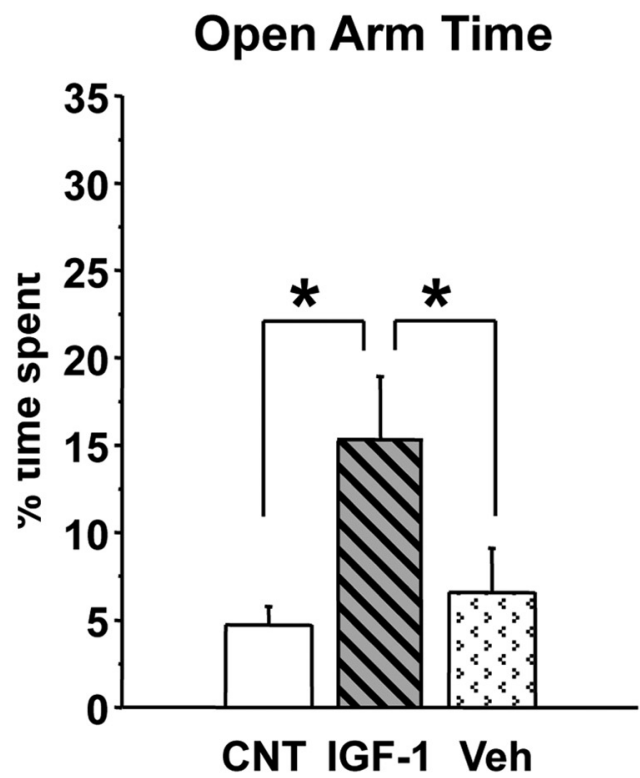

C

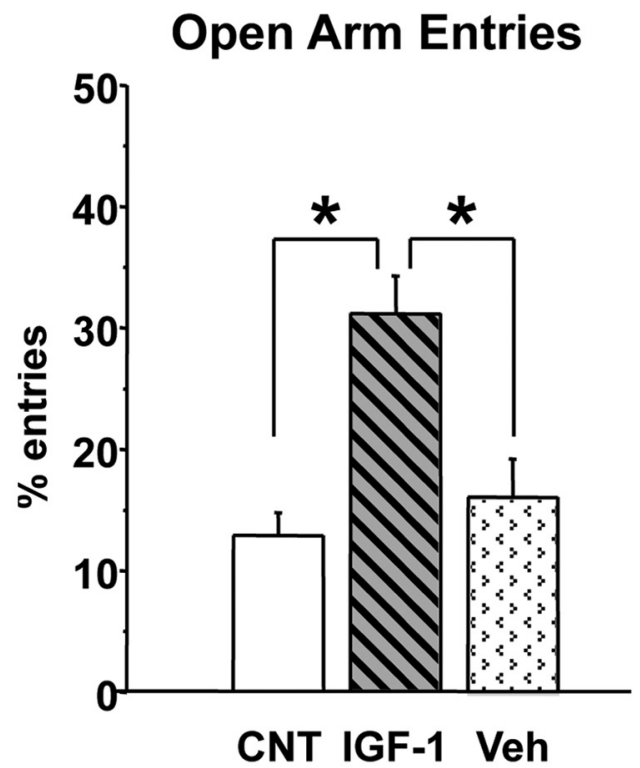

D1

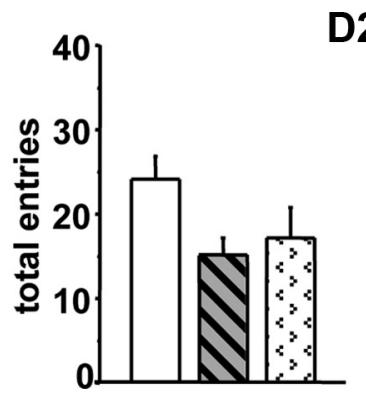

D2

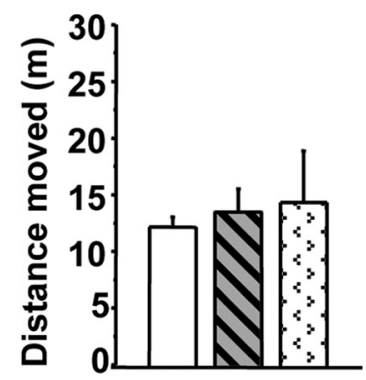

Figure 2. Early postnatal IGF-1 subcutaneous administration decreases adult anxiety-like behavior. $\boldsymbol{A}$, Experimental protocol. $\boldsymbol{B}, \boldsymbol{C}$, Mean percentage of time spent in the open arms ( $\boldsymbol{B})$ and mean percentage of entries in the open arms $(\boldsymbol{C})$ at $\mathrm{P} 60$ for IGF-1-treated, Veh-treated, and

post hoc Dunn's method; percentage open arm entries, one-way ANOVA, $\mathrm{df}=2, p<0.001(F=13.212)$, post hoc Holm-Sidak method]. The absence of effects of Veh injections suggests that reduced anxiety-like behavior in IGF-1-treated rats cannot be simply ascribed to the manipulations involved in the injection procedures. Both IGF-1 and Veh treatment did not influence the total number of arm entries or total distance moved (Fig. $2 D 1, D 2)$ [one-way ANOVA, $\mathrm{df}=2$; total entries, $p>0.05(F=$ $2.629)$; total distance moved, $p>0.05(F=0.324)]$.

The reduction of anxiety response elicited by IGF-1 treatment was comparable with that produced by EE or massage; indeed, the performance of IGF-1-treated rats did not differ from that of MSG, EE-P12, EE-P45, or EE-P60 rats [one-way ANOVA, df = 4 ; percentage open arm time, $p>0.05(F=0.112)$; percentage open arm entries, $p>0.05(F=2.041)]$.

Thus, an increase in circulating IGF-1 during the first postnatal days is sufficient to produce long-term effects on anxiety-like behavior.

Molecular mechanisms underlying early enriched experience and IGF-1 action on anxiety-like behavior

To test whether early increases in IGF-1, either after enriched experience or direct IGF-1 administration, might modify cellular responsiveness to IGF-1, we measured IGF-1R levels in the hippocampus by Western blot, both during development (P12) and in adulthood (P60) in IGF-1-treated (P12, $n=6$; P60, $n=6)$ and EE-P45 (P12, $n=7$; P60, $n=9$ ) animals. IGF-1R levels were transiently increased in IGF-1-treated animals (P12, $134.7 \pm 6.6$; P60, $110 \pm 9.6)$ compared with CNT $(\mathrm{P} 12, n=6,102.8 \pm 8.2$; $\mathrm{P} 60, n=7,98.2 \pm 4.7$ ), with increase being significant at P12 but not P60. A long-lasting IGF-1R increase was found in EE animals $(\mathrm{P} 12,149.1 \pm 9.1 ; \mathrm{P} 60,123 \pm 5.9 ;$ Fig. $3 A, D)$ [one-way ANOVA, $\mathrm{df}=3$; mean IGF-1R level at P12, $p<0.01(F=6.091)$; mean IGF-1R level at P60, $p<0.05(F=4.96)$, post hoc Holm-Sidak method]. To control for possible aspecific effects of subcutaneous injections, a group of saline-treated animals was also analyzed (P12, $n=4,115.0 \pm 10.6 ; \mathrm{P} 60, n=7,92.3 \pm 13.0)$ : no difference with CNT animals was found (Fig. $3 A, D$ ). This confirms that IGF-1 effects on anxiety-like behavior cannot be ascribed to the manipulations associated with the treatment [one-way ANOVA, df $=3$; mean IGF-1R level at P12, $p<0.01$ $(F=6.091)$; mean IGF-1R level at P60, $p<0.05(F=4.96)$, post hoc Holm-Sidak method].

To investigate the molecular mechanisms underlying the long-lasting effects of early enrichment of experience and of IGF-1 in reducing adult anxiety-like behavior, we assessed the hippocampal expression of GRs in EE and IGF-1-treated animals. Increased hippocampal GRs is thought to mediate the action of early positive experiences such as high levels of maternal care or handling on adult anxiety-like behavior (Meaney and Szyf, 2005). We measured GR expression by Western blot at both P12 and P60 in the same protein extracts used for assessing IGF-1R to test for effects during development and in the adult. We found that GR expression was not affected by treatments at P12, but notably it was at P60; indeed, hippocampal GR expression in EE and IGF1-treated animals was significantly higher than in CNT rats

CNT rats. CNT and Veh groups differ from IGF-1-treated group (asterisks, $p<0.05$ ); the former two groups do not differ (one-way ANOVA). Vertical bars are SEM. D1, D2, Mean total arm entries (D1) and mean distance moved (D2) during the test for all experimental groups. No difference was found between groups (one-way ANOVA). Vertical bars are SEM. 


\section{P12 hippocampus}

A
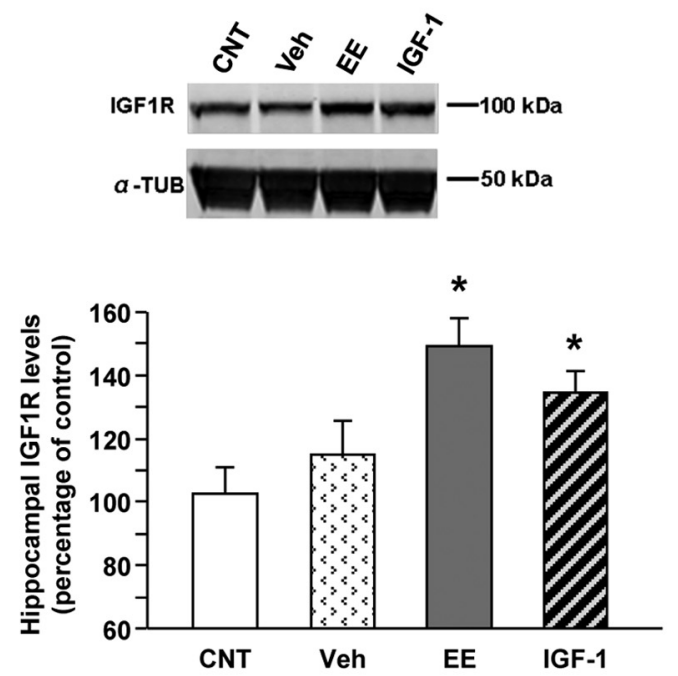

B
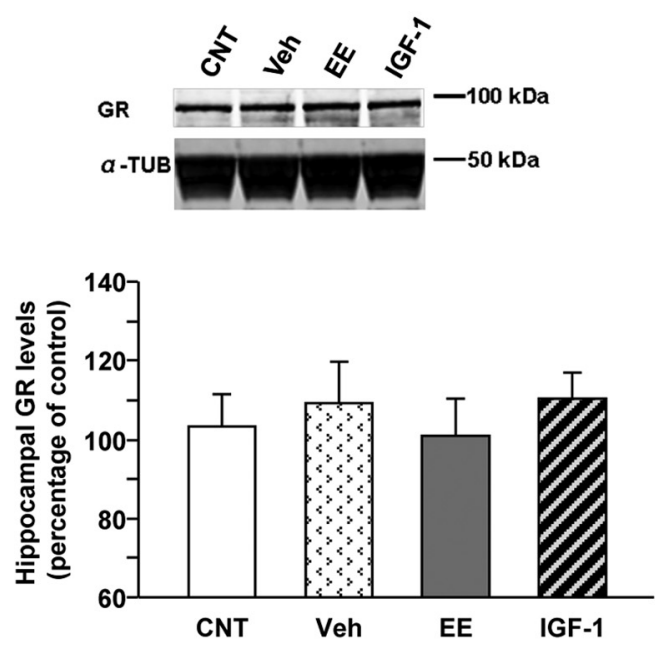

C
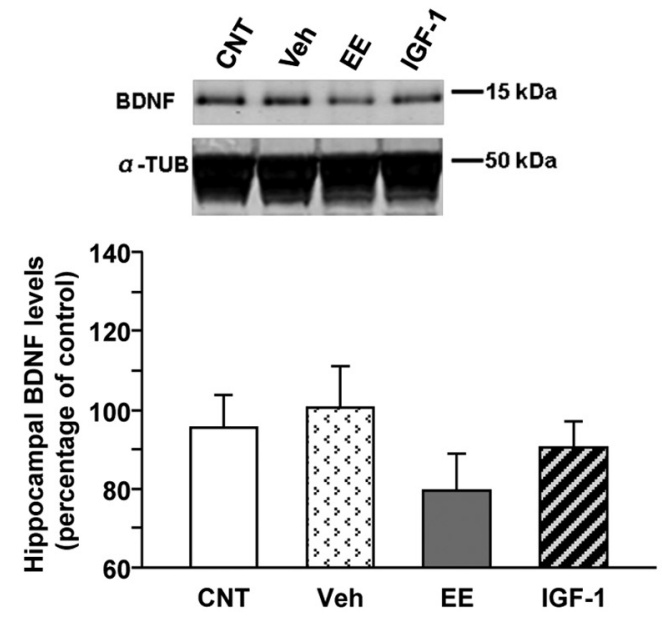

\section{P60 hippocampus}
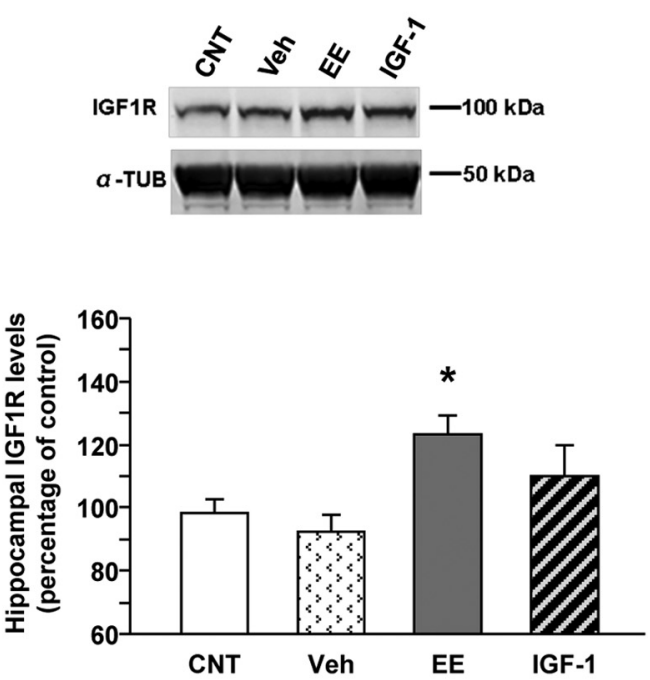

E
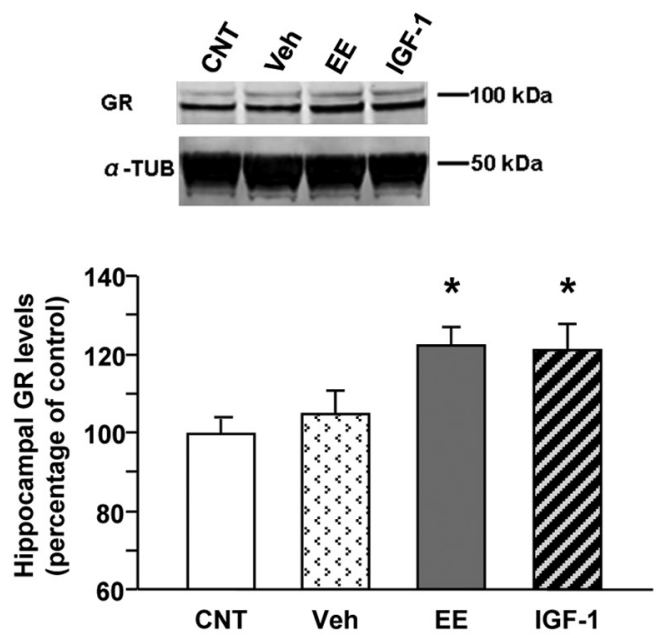

$\mathbf{F}$

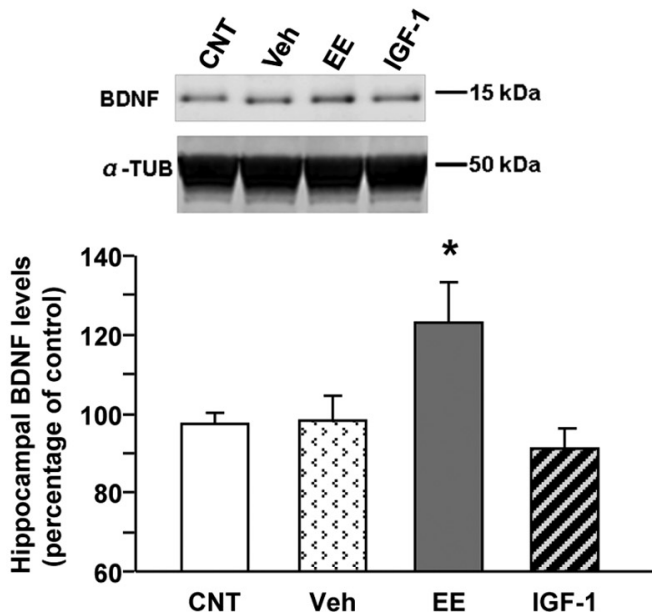

Figure 3. Molecular mechanisms underlying EE and IGF-1 action on anxiety-like behavior: Western blot analysis of hippocampal IGF-1R, GR, and BDNF levels at P12 and P60. In all panels, the inset is a representative immunoblotting showing the corresponding protein levels in the hippocampus of animals in the different experimental groups [animals reared in standard conditions (CNT), systemically injected with saline (Veh), reared in enriched conditions up to P45 (EE), and reared in standard conditions but subcutaneously treated with IGF-1 (IGF-1)]. $\alpha$-Tubulin ( $\alpha$-TUB) is the internal standard. $A-F$, Quantification of protein levels; data aremean \pm SEM. Asterisks denote significant differences $(p<0.05)$. $A$, Quantification of IGF-1Rlevels at P12. In animals reared in EE conditions and in those treated with IGF-1, the amount of IGF-1R is significantly higher than in CNT animals, whereas saline injections have no effects (one-way ANOVA). B, Quantification of GR levels at P12. At this developmental stage, there is no difference among experimental groups (one-way ANOVA). C, Quantification of BDNF levels at P12. No difference among experimental groups is detectable (one-way ANOVA). D, Quantification of IGF-1R levels at P60. In animals reared in EE conditions, the hippocampal expression of IGF-1R is significantly higher than in CNT animals (one-way ANOVA). E, Quantification of GR levels at P60. In animals reared in EE conditions and in those treated with IGF-1, the hippocampal expression of GR is significantly higher than in CNT animals, whereas saline injections do not affect the amount of GR protein (one-way ANOVA). $F$, Quantification of BDNF levels at P60. In animals reared in EE conditions, the hippocampal expression of BDNF is significantly higher than in CNT animals (one-way ANOVA). 
(CNT, $99.7 \pm 3.8$; EE, $122.3 \pm 4.7$; IGF-1, $121.0 \pm 6.7$; Fig. 3 B, E). No effect was detected in Veh-treated animals (Veh, $104.9 \pm 6.0$ ) [one-way ANOVA, $\mathrm{df}=3$; mean GR level at P12, $p>0.05(F=$ $0.584)$; mean GR level at P60, $p<0.05$ $(F=4.712)$, post hoc Holm-Sidak method].

BDNF is known to be precociously increased in the brain by high levels of maternal care (Liu et al., 2000) and early enriched experience (Cancedda et al., 2004) and is potentially related to the long-term effects of high level of maternal care on adult anxiety behavior (Liu et al., 2000). We have assessed hippocampal BDNF levels by means of Western blot, finding no significant effects at P12 but a significant increase in EE animals at P60 (CNT, 97.4 \pm 2.6; Veh, $98.3 \pm 6.3$; EE, $123.0 \pm 10.3$; IGF-1, $91.2 \pm 5.2$; Fig. $3 C, F)$ [one-way ANOVA, $\mathrm{df}=3$; mean BDNF level at P12, $p>0.05(F=0.733)$; mean BDNF level at P60, $p<0.05(F=$ 4.018), post hoc Holm-Sidak method].

Thus, both early enriched experiences and IGF-1 administration restricted to the period $\mathrm{P} 1-\mathrm{P} 9$ are sufficient to program the HPA axis toward a more active feedback control and possibly a more adaptive response in the adult.

\section{Block of early IGF-1 prevents massage and enrichment effects on anxiety-like behavior}

To assess whether IGF-1 is a crucial factor mediating long-term effects of EE and massage on anxiety-like behavior, we blocked IGF-1 action by means of systemic JB1 injections from P1 to P9 in EE-P45 $(n=16)$ and MSG $(n=16)$ rats. We decided to assess the effects of blocking circulating IGF-1 in rats enriched up to P45 to strengthen the hypothesis that IGF-1 response to early environmental enrichment is crucial to produce long-term effects on anxiety-like behavior.

As shown in Figure 4, the percentage of time spent in open arms or open arm entries for JB1-treated MSG (percentage time in open arms, MSG $+\mathrm{JB} 1=1.27 \pm 0.47$; percentage open arm entries, MSG + JB1 = 14.22 \pm 2.40 ) or EE (percentage time in open arms, $\mathrm{EE}+\mathrm{JB} 1=7.70 \pm 2.08$; percentage open arm entries, $\mathrm{EE}+\mathrm{JB} 1=15.39 \pm 3.05)$ animals did not significantly differ from that of CNT rats $(n=31)$ and was significantly lower than in MSG $(n=5$, percentage time in open arms, MSG + Veh $=19.32 \pm 6.94$; percentage open arm entries, MSG + Veh $=36.86 \pm 9.37)$ and enriched $(n=13$, percentage time in open arms, $\mathrm{EE}+\mathrm{Veh}=20.68 \pm 3.98$; percentage open arm entries, $\mathrm{EE}+\mathrm{Veh}=31.36 \pm 4.45)$ animals treated with Veh [one-way ANOVA, $\mathrm{df}=4$; percentage time in open arms, $p<0.001(F=12.97)$; percentage open arm entries, $p<0.001(F=8.717)$, post hoc Holm-Sidak method]. Performance in EE or MSG animals treated with Veh did not differ from that of the respective untreated group. Both total arm entries and total distance moved were comparable between the experimental groups (Fig. $4 D 1, D 2$ ) [oneway ANOVA, $\mathrm{df}=4$; total entries, $p>0.05(F=2.456)$; total distance moved, $p>0.05(F=0.0561)]$. These findings allow ruling out that the effects of the treatments on anxiety-like responses were dependent on low level of activity in the maze.

To assess whether JB1 treatment in EE animals might have affected gross brain development, we determined neuronal density in a number of structures (hippocampus, amygdala, and visual cortex) in a group of P18 EE animals treated with either Veh or JB1 ( $n=4$ for each group). We confirmed the results obtained by Ciucci et al. (2007) for the visual cortex with a protocol of later JB1 infusion, finding no significant effect of JB1 treatment (EE + Veh $=3163 \pm 306$ neurons/ $\mathrm{mm}^{2} ; \mathrm{EE}+\mathrm{JB} 1=3305 \pm 239$ neurons $/ \mathrm{mm}^{2} ; t$ test, $\left.p>0.05\right)$. We also found that neuronal density in two structures participating in the anxiety response did not differ between EE animals treated with Veh or JB1 (hippocampus: CA1-C3, EE + Veh $=1987 \pm 34$ neurons $/ \mathrm{mm}^{2}, \mathrm{EE}+\mathrm{JB} 1=2076 \pm 35$ neurons $/ \mathrm{mm}^{2} ; t$ test, $p>0.05 ; \mathrm{DG}, \mathrm{EE}+\mathrm{Veh}=3145 \pm 178$ neurons $/ \mathrm{mm}^{2}, \mathrm{EE}+\mathrm{JB} 1=3315 \pm 152$ neurons $/ \mathrm{mm}^{2} ; t$ test, $p>0.05$; amygdala: $\mathrm{EE}+\mathrm{Veh}=2609 \pm 90$ neurons $/ \mathrm{mm}^{2}$, $\mathrm{EE}+\mathrm{JB} 1=2792 \pm 148$ neurons $/ \mathrm{mm}^{2}$; Mann-Whitney ranksum test, $p>0.05)$. The lack of difference in neuronal density between two groups showing a strong difference in anxietylike behavior, such as EE and EE + JB1 rats, suggests that gross differences in cell number are not involved in the different behavioral effects we observed.

Thus, antagonizing IGF- 1 action in the first week of life completely prevents EE and massage effects on anxiety-like behavior in adult animals, supporting the idea that early interactions with the environment might program the stress response and that IGF-1 action is a necessary mediator of this programming.

\section{Discussion}

It is already well known that early life experiences can affect brain development, contributing to shape interindividual dif- 
ferences in vulnerability to stress and anxiety-like behavior. The importance of early experiences in causing long-lasting effects in the offspring has been shown in both human and animal models (Wilson 2003; Meaney and Szyf, 2005; Zhang et al., 2013).

We have shown here that early enrichment of experience by means of EE or massage causes a reduction in the anxiety-like behavior that is evident in adult animals and that IGF-1 level in the first days of life is a crucial factor in mediating these longlasting effects. We have not examined animals at later ages, so we cannot say whether the effects documented in young adults are also present at later adulthood stages or even in aged subjects. However, the temporal distance between the end of the treatments and the day of testing allows us to say that both early experiences produced long-lasting effects on anxiety-like behavior. Both early enrichment and IGF-1 administration produce a strong increase in adult hippocampal GR level. This suggests a role for IGF-1 in programming the adult anxiety response exerted by early life experiences.

\section{Early massage and EE decrease anxiety-like behavior in adult rat via IGF-1}

Our results show that a programming of adult anxiety responses can be observed not only in pups born to mothers exhibiting higher levels of maternal care or subjected to handling (for review, see Meaney and Szyf, 2005) but also in pups born into an EE or subjected to massage. It is likely that early $\mathrm{EE}$ is effective because it causes an increase in the level of maternal care (Sale et al., 2004) and that massage is effective because it mimics the tactile warm stimulation provided by the mother's licking and grooming. However, it is the first time that a direct link between EE or massage and adult anxiety-like behavior is provided. It is particularly interesting that massage or EE restricted to the first $12 \mathrm{~d}$ of life of rat pups cause a decrease in the anxiety-like behavior, tested at P60.

Our data demonstrate that IGF-1 is involved in mediating the long-term effects of early massage and EE on anxiety-like behavior. Indeed, subcutaneous injections of IGF-1 from P1 to $\mathrm{P} 9$ in rat pups reproduced the effects of massage and EE protocols. Because Veh treatment did not modify adult performance in the EPM, we can conclude that IGF-1 effects are not attributable to the pup manipulations needed for subcutaneous injections. In addition, subcutaneous injections from P1 to P9 of IGF-1R antagonist JB1 blocked massage and EE effects. Therefore, these results demonstrate for the first time that early enriched experiences may affect anxiety-like behavior in the adult through the IGF-1 pathway, suggesting an IGF-1 programming action on the adult ability to cope with stressful situations.

We think that the effects of our IGF- 1 and JB- 1 subcutaneous treatments derive from the fact that they increase and reduce, respectively, the quantity of circulating IGF-1 crossing the blood-brain barrier (Aleman and Torres-Alemán, 2009; Fernandez and Torres-Alemán, 2012), thus increasing or reducing IGF-1 availability in the brain. It is known that, even during development, IGF-1 found in the brain is predominantly coming from peripherally produced serum IGF-1 (Sale et al., 2007; Aleman and Torres-Alemán, 2009). Indeed, as shown by Guzzetta et al. (2009), subcutaneous administration of JB1 to MSG rat pups, done with exactly the same dosage and protocol we used, com- pletely prevents massage, which increases IGF-1 in both the blood and brain, from accelerating visual acuity development.

\section{Mechanisms of action}

The results of the analysis of IGF-1R suggest that, at first, IGF-1 enhances its own effectiveness through the increase in the expression of IGF-1R. This effect is evident at P12 in both EE and IGF-1-treated animals. The fact that IGF-1R increase is no longer evident in adult animals for IGF-1 treatment whereas treatment effect is evident behaviorally and in terms of hippocampal GR expression suggests that it is the early increase in IGF-1R that is crucial to trigger both the reduction in adult anxiety and the enhancement of HPA axis feedback. The increase in IGF-1R found at P60 in EE animals (EE up to P45) is probably attributable to the extension of enriched experience, but this sustained increase does not produce an advantage in terms of either behavioral performance or GR hippocampal expression. To reinforce this interpretation, block of IGF-1 between P1 and P9 is sufficient to completely delete the effects of enriched early experience on anxiety-like behavior. To better understand the implications of the early increase in IGF-1R, it will be important to determine which cell types upregulate IGF-1R. Indeed, IGF-1R is widely expressed in all cell types in the brain, starting from early ages (Bondy, 1991; Yan et al., 2011; Fernandez and Torres-Alemán 2012).

How does the early enhancement in IGF-1 system translate in a long-term reduction in anxiety-like behavior? The literature suggested that levels of adult hippocampal GRs, which regulate the HPA response to stress through a negative feedback, are sensitive to early experience, such as handling and high maternal care levels, and correlate with anxiety-like behavior and stress responsiveness in the adult (Weaver et al., 2004, van Hasselt et al., 2012; for review, see Zhang et al., 2013).

Our data on GR expression show indeed that hippocampal GR level is higher at P60 in enriched and IGF-1-treated rats, validating the hypothesis of an action of IGF-1 on anxiety behavior in the adult via increased GR expression. We have not tested GR expression in MSG rats, but it might be hypothesized that massage, which is a tactile stimulation protocol mimicking maternal licking and grooming and that increases early IGF-1 levels, might act on adult anxiety-like behavior through a higher hippocampal GR expression. Of particular relevance is the fact that IGF-1 treatment, restricted to the early pre-weaning period, is sufficient to trigger an increase in hippocampal GRs evident in adult age.

How can early enriched experience or IGF-1 exert long-term effects on hippocampal GR expression? A possible molecular candidate is BDNF. BDNF expression is increased after IGF-1 treatment both in the adult (Carro et al., 2000) and during development (Landi et al., 2009), and several studies suggested a correlation between early increase in hippocampal BDNF mRNA, enhanced levels of adult hippocampal GR, and reduced adult anxiety-like behavior in the offspring of mothers that show high levels of licking and grooming (Liu et al., 2000; Meaney and Szyf, 2005). Conversely, maternal separation, which reduces hippocampal BDNF both in the adult and during development (Roceri et al., 2002; Kuma et al., 2004), also reduces adult GR expression (Navailles et al., 2010). Early enriched experience could increase, through IGF-1, BDNF levels in the brain, thus affecting hippocampal GR expression and stress response in adult rats. This hypothesis is not supported by our data. Indeed, BDNF levels are not increased by enrichment or IGF-1 during development, and the increase in the adult, detectable only in enriched 
animals, does not seem to be crucial for the behavioral phenotype because both IGF-1 and enrichment equally reduce adult anxiety. However, it has to be noted that the effect of early enrichment on hippocampal BDNF during development is transient, being detected at P8 and absent at P18 and P90 (Liu et al., 2000), so the peak of BDNF increase in enriched animals might occur at earlier ages than the one we examined (P12).

Therefore, the pathway between IGF-1 and GRs needs additional investigation, in terms of the involvement of other molecular factors, such as serotonin, which is increased by both early enriched experiences (for review, see Hackman et al., 2010) and IGF-1 (Hoshaw et al., 2008).

\section{Conclusion}

The present results suggest that environmentally determined early increase in IGF-1 is a key factor in mediating the longlasting effects of early postnatal experiences on anxiety-like behavior. A deeper understanding of the consequences of early experience variations and in particular of the underlying mechanisms of action on the development of brain and behavior has an important clinical relevance. It may potentially allow designing interventions to prevent or rescue the negative effects of adverse early experiences, reducing the risk for pathological disorders.

\section{References}

Aleman A, Torres-Alemán I (2009) Circulating insulin-like growth factor I and cognitive function: neuromodulation throughout the lifespan. Prog Neurobiol 89:256-265. CrossRef Medline

Bondy CA (1991) Transient IGF-I gene expression during the maturation of functionally related central projection neurons. J Neurosci 11:3442-3455. Medline

Brock RS, Gebrekristos BH, Kuniyoshi KM, Modanlou HD, Falcao MC, Beharry KD (2011) Biomolecular effects of JB1 (an IGF-I peptide analog) in a rat model of oxygen-induced retinopathy. Pediatr Res 69:135-141. CrossRef Medline

Cancedda L, Putignano E, Sale A, Viegi A, Berardi N, Maffei L (2004) Acceleration of visual system development by environmental enrichment. J Neurosci 24:4840-4848. CrossRef Medline

Carro E, Nuñez A, Busiguina S, Torres-Aleman I (2000) Circulating insulinlike growth factor I mediates effects of exercise on the brain. J Neurosci 20:2926-2933. Medline

Chapillon P, Patin V, Roy V, Vincent A, Caston J (2002) Effects of pre- and postnatal stimulation on developmental, emotional, and cognitive aspects in rodents: a review. Dev Psychobiol 41:373-387. CrossRef Medline

Chatterjee D, Chatterjee-Chakraborty M, Rees S, Cauchi J, de Medeiros CB, Fleming AS (2007) Maternal isolation alters the expression of neural proteins during development: "stroking" stimulation reverses these effects. Brain Res 1158:11-27. CrossRef Medline

Chee SS, Menard JL (2011) Lesions of the dorsal lateral septum do not affect neophagia in the novelty induced suppression of feeding paradigm but reduce defensive behaviours in the elevated plus maze and shock probe burying tests. Behav Brain Res 220:362-366. CrossRef Medline

Ciucci F, Putignano E, Baroncelli L, Landi S, Berardi N, Maffei L (2007) Insulin-like growth factor 1 (IGF-1) mediates the effects of enriched environment (EE) on visual cortical development. PLoS One 2:e475. CrossRef Medline

Fernandez AM, Torres-Alemán I (2012) The many faces of insulin-like peptide signalling in the brain. Nat Rev Neurosci 13:225-239. CrossRef Medline

Fernandez AM, Garcia-Estrada J, Garcia-Segura LM, Torres-Aleman I (1997) Insulin-like growth factor I modulates c-Fos induction and astrocytosis in response to neurotoxic insult. Neuroscience 76:117-122. CrossRef Medline

Field T, Diego M, Hernandez-Reif M, Dieter JN, Kumar AM, Schanberg S, Kuhn C (2008) Insulin and insulin-like growth factor-1 increased in preterm neonates following massage therapy. J Dev Behav Pediatr 29:463466. CrossRef Medline

Francis DD, Diorio J, Plotsky PM, Meaney MJ (2002) Environmental en- richment reverses the effects of maternal separation on stress reactivity. J Neurosci 22:7840-7843. Medline

Guzzetta A, Baldini S, Bancale A, Baroncelli L, Ciucci F, Ghirri P, Putignano E, Sale A, Viegi A, Berardi N, Boldrini A, Cioni G, Maffei L (2009) Massage accelerates brain development and the maturation of visual function. J Neurosci 29:6042-6051. CrossRef Medline

Hackman DA, Farah MJ, Meaney MJ (2010) Socioeconomic status and the brain: mechanistic insights from human and animal research. Nat Rev Neurosci 11:651-659. CrossRef Medline

Hayton SJ, Mahoney MK, Olmstead MC (2012) Behavioral traits predicting alcohol drinking in outbred rats: an investigation of anxiety, novelty seeking, and cognitive flexibility. Alcohol Clin Exp Res 36:594-603. CrossRef Medline

Hoshaw BA, Hill TI, Crowley JJ, Malberg JE, Khawaja X, Rosenzweig-Lipson S, Schechter LE, Lucki I (2008) Antidepressant-like behavioral effects of IGF-I produced by enhanced serotonin transmission. Eur J Pharmacol 594:109-116. CrossRef Medline

Kuma H, Miki T, Matsumoto Y, Gu H, Li HP, Kusaka T, Satriotomo I, Okamoto H, Yokoyama T, Bedi KS, Onishi S, Suwaki H, Takeuchi Y (2004) Early maternal deprivation induces alterations in brain-derived neurotrophic factor expression in the developing rat hippocampus. Neurosci Lett 372:68-73. CrossRef Medline

Landi S, Ciucci F, Maffei L, Berardi N, Cenni MC (2009) Setting the pace for retinal development: environmental enrichment acts through insulin-like growth factor 1 and brain-derived neurotrophic factor. J Neurosci 29: 10809-10819. CrossRef Medline

Laviola G, Rea M, Morley-Fletcher S, Di Carlo S, Bacosi A, De Simone R, Bertini M, Pacifici R (2004) Beneficial effects of enriched environment on adolescent rats from stressed pregnancies. Eur J Neurosci 20:16551664. CrossRef Medline

Levine S (1967) Maternal and environmental influences on the adrenocortical response to stress in weanling rats. Science 156:258-260. CrossRef Medline

Liu D, Diorio J, Tannenbaum B, Caldji C, Francis D, Freedman A, Sharma S, Pearson D, Plotsky PM, Meaney MJ (1997) Maternal care, hippocampal glucocorticoid receptors, and hypothalamic-pituitary-adrenal responses to stress. Science 277:1659-1662. CrossRef Medline

Liu D, Diorio J, Day JC, Francis DD, Meaney MJ (2000) Maternal care, hippocampal synaptogenesis and cognitive development in rats. Nat Neurosci 3:799-806. CrossRef Medline

Malberg JE, Platt B, Rizzo SJ, Ring RH, Lucki I, Schechter LE, RosenzweigLipson S (2007) Increasing the levels of insulin-like growth factor-I by an IGF binding protein inhibitor produces anxiolytic and antidepressantlike effects. Neuropsychopharmacology 32:2360-2368. CrossRef Medline

Meaney MJ, Szyf M (2005) Environmental programming of stress responses through DNA methylation: life at the interface between a dynamic environment and a fixed genome. Dialogues Clin Neurosci 7:103-123. Medline

Mohammed AH, Henriksson BG, Söderström S, Ebendal T, Olsson T, Seckl JR (1993) Environmental influences on the central nervous system and their implications for the aging rat. Behav Brain Res 57:183-191. CrossRef Medline

Navailles S, Zimnisky R, Schmauss C (2010) Expression of glucocorticoid receptor and early growth response gene 1 during postnatal development of two inbred strains of mice exposed to early life stress. Dev Neurosci 32:139-148. CrossRef Medline

Olsson T, Mohammed AH, Donaldson LF, Henriksson BG, Seckl JR (1994) Glucocorticoid receptor and NGFI-A gene expression are induced in the hippocampus after environmental enrichment in adult rats. Brain Res Mol Brain Res 23:349-353. Medline

Roceri M, Hendriks W, Racagni G, Ellenbroek BA, Riva MA (2002) Early maternal deprivation reduces the expression of BDNF and NMDA receptor subunits in rat hippocampus. Mol Psychiatry 7:609-616. CrossRef Medline

Sale A, Putignano E, Cancedda L, Landi S, Cirulli F, Berardi N, Maffei L (2004) Enriched environment and acceleration of visual system development. Neuropharmacology 47:649-660. CrossRef Medline

Sale A, Cenni MC, Ciucci F, Putignano E, Chierzi S, Maffei L (2007) Maternal enrichment during pregnancy accelerates retinal development of the fetus. PLoS One 2:e1160. CrossRef Medline

Sale A, Berardi N, Maffei L (2009) Enrich the environment to empower the brain. Trends Neurosci 32:233-239. CrossRef Medline 
Schanberg SM, Field TM (1987) Sensory deprivation stress and supplemental stimulation in the rat pup and preterm human neonate. Child Dev 58:1431-1447. CrossRef Medline

Trent NL, Menard JL (2011) Infusions of neuropeptide Y into the lateral septum reduce anxiety-related behaviors in the rat. Pharmacol Biochem Behav 99:580-590. CrossRef Medline

van Hasselt FN, Cornelisse S, Zhang TY, Meaney MJ, Velzing EH, Krugers HJ, Joëls M (2012) Adult hippocampal glucocorticoid receptor expression and dentate synaptic plasticity correlate with maternal care received by individuals early in life. Hippocampus 22:255-266. CrossRef Medline

Weaver IC, Diorio J, Seckl JR, Szyf M, Meaney MJ (2004) Early environmental regulation of hippocampal glucocorticoid receptor gene expression: characterization of intracellular mediators and potential genomic target sites. Ann N Y Acad Sci 1024:182-212. CrossRef Medline

Wilkin MM, Waters P, McCormick CM, Menard JL (2012) Intermittent physical stress during early- and mid-adolescence differentially alters rats' anxietyand depression-like behaviors in adulthood. Behav Neurosci 126:344-360. CrossRef Medline
Wilson SL (2003) Post-institutionalization: the effects of early deprivation on development of Romanian adoptees. Child Adolesc Social Work J 20:473-483. CrossRef

Witty CF, Gardella LP, Perez MC, Daniel JM (2013) Short-term estradiol administration in aging ovariectomized rats provides lasting benefits for memory and the hippocampus: a role for insulin-like growth factor-I. Endocrinology 154:842-852. CrossRef Medline

Yan H, Mitschelen M, Bixler GV, Brucklacher RM, Farley JA, Han S, Freeman WM, Sonntag WE (2011) Circulating IGF1 regulates hippocampal IGF1 levels and brain gene expression during adolescence. J Endocrinol 211: 27-37. CrossRef Medline

Zhang TY, Labonté B, Wen XL, Turecki G, Meaney MJ (2013) Epigenetic mechanisms for the early environmental regulation of hippocampal glucocorticoid receptor gene expression in rodents and humans. Neuropsychopharmacology 38:111-123. CrossRef Medline 